\title{
Incidencia y mortalidad de bacteriemia en un hospital clínico docente en Santiago de Chile
}

\author{
MARÍA GAMBRA ${ }^{1}$, JEFERSON FLORES ${ }^{2, a}$, \\ KATHLEEN RAMÍREZ ${ }^{\mathrm{b}}$, SOFÍA PALMA ${ }^{1}$, PEDRO ZITKO ${ }^{4}$, \\ MARÍA TERESA VALENZUELA ${ }^{3}$, CARLOS BELTRÁN ${ }^{1}$
}

\section{Incidence and mortality of bacteremia in a public hospital in Santiago}

Background: The incidence of bacteremia has increased over the last decade due to the aging of the population and the wide implementation of invasive nosocomial procedures and wide-spectrum antimicrobial treatments. Aim: To assess the epidemiology of hospital and ambulatory bacteremias in a public hospital in Santiago. Material and Methods: A prospective longitudinal cohort study of laboratory-confirmed adult patients with bacteremia was undertaken at a public hospital in Santiago, between June 1, 2007 and April 30, 2008. Demographic, clinical, and laboratory data was collected into a standardized study-specific form. Results: In the study period, 253 cases of true bacteremia were identified, with an incidence of 11 per 1,000 patient discharges (63.6\% nosocomial, 36.7\% fatal). Only 2/3 of the cohort was alive at day 28 of hospitalization. Variables associated with mortality were age with a hazard ratio (HR) of 2.31; (95\% confidence intervals (CI) 1.42-3.77); female gender (HR, 1.70; 95\% CI 1.06- 2.71); shock (HR, 3.24; 95\%CI 2.01- 5.22); and C reactive protein (HR, 2.10; 95\% CI 1.17- 3.78). The variable associated with lower mortality was surgery (HR, 0.43; 95\% CI 0.25-0.75). Selective empiric treatment did not improve survival. Conclusions: Besides age and gender, survival can be influenced by modifiable variables such as presence of shock and surgical intervention, which may provide an opportunity to improve outcomes.

(Rev Med Chile 2012; 140: 859-866).

Key words: Bacteremia; Epidemiology; Risk factors; Survival.

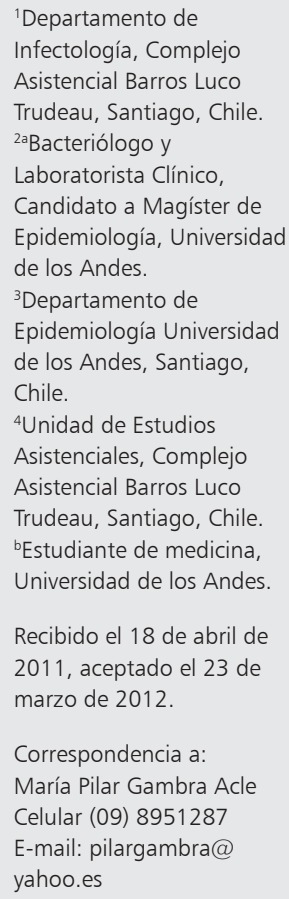

A pesar de todos los avances en terapia y soporte de pacientes, la bacteriemia continúa siendo una causa importante de morbilidad y mortalidad ocasionando 150.000 muertes anuales en Europa y 210.000 muertes anuales en Estados Unidos de Norteamérica ${ }^{1}$. La incidencia de bacteriemia ha ido en aumento, probablemente gatillado por el envejecimiento de la población, el incremento de procedimientos invasivos, uso de antibióticos de amplio espectro, tratamientos inmunodepresores y quimioterapia ${ }^{2}$. Actualmente, se estima una incidencia de bacteriemia y fungemia entre 3 y 28 episodios por 1.000 ingresos hospita- $\operatorname{larios}^{3-7}$. Un tercio de las bacteriemias aisladas en el hospital son de origen comunitario ${ }^{8}$.

La mortalidad reportada en bacteriemia adquirida en la comunidad es de 10,2\%-13\%; cuando la bacteriemia se asocia a cuidados sanitarios, es de $18-20 \%$ y en aquella adquirida al interior del hospital es de $23-30 \%{ }^{9,10}$. Los factores asociados a muerte más relevantes han sido la adquisición nosocomial, la edad superior a 65 años, presencia de shock, el origen urinario y pulmonar, la aparición de coagulación intravascular diseminada, enfermedad grave de base (neoplasia o neutropenia), tratamiento antibiótico inapropiado e internación 
en unidad de cuidados intensivos ${ }^{2,11-16}$. No se ha logrado demostrar que las bacteriemias por agentes resistentes a múltiples antimicrobianos produzcan un exceso de muertes ${ }^{17,18}$.

Inicialmente, entre 1979 y 1987 las bacterias Gram negativas fueron los agentes predominantes de sepsis intrahospitalaria, mientras que en años subsecuentes las bacterias Gram positivas fueron los microorganismos predominantes ${ }^{19}$. Es importante en este setting la relevancia que adquiere Klebsiella spp, Pseudomona aeruginosa y Acinetobacter baumanii. Al mismo tiempo se ha constatado un aumento de aislamiento de Candida spp de 8\% en 1995 a $12 \%$ en $2002^{20}$. En bacteriemias extrahospitalarias, bacterias Gram negativas son más prevalentes, guardando íntima relación con los focos clínicos que frecuentemente las originan $^{19,21,22}$.

En Chile, desde 1983 se vigila activamente las infecciones del torrente sanguíneo (ITS), las asociadas a catéteres venoso central (CVC), a nutrición parenteral y a catéteres umbilicales en niños. A partir del año 2006 se vigilan las bacteriemias asociados a catéteres de hemodiálisis. De esta vigilancia se desprende Staphylococcus aureus como el agente más frecuentemente aislado, seguido por Staphylococcus coagulasa negativa. El género Candida spp ha mostrado un aumento porcentual dentro de los agentes aislados ITS/CVC en servicio de adultos de los últimos 5 años $(2,8 \%$ en 2002 a $5,8 \%$ en 2006 $)^{23}$.

Los datos existentes en Chile reflejan una parte de la realidad, la hospitalaria y dentro de ella, la asociada a procedimientos que generan mayor riesgo de bacteriemia. La dinámica epidemiológica es particular dentro un mismo hospital y entre diferentes centros de atención de salud ${ }^{24}$. Existen pocos estudios que investiguen los factores asociados a la ocurrencia de la muerte atribuida a bacteriemia y menos aun a la sobrevida de estos pacientes ${ }^{12,15,25}$.

El objetivo de este estudio fue establecer la incidencia de bacteriemia de pacientes atendidos en el Complejo Asistencial Barros Luco Trudeau (CABLT) y los factores clínicos que afectan la sobrevida intrahospitalaria.

\section{Materiales y Métodos}

\section{Lugar y tipo de estudio}

Se realizó un estudio observacional de cohorte prospectivo en CABLT, el cual es un hospital do- cente asistencial ubicado en el sur de la ciudad de Santiago de Chile. Tiene 600 camas y la población asignada es de 1.000.000 de personas. Este estudio se realizó entre el 1 de junio de 2007 y 30 de abril de 2008 .

\section{Criterios de inclusión}

Se incluyó pacientes de 15 años y más que ingresaron a CABLT entre 1 de junio de 2007 a 30 de abril de 2008 con hemocultivo positivos y cumplieron con la definición de bacteriemia verdadera $^{26}$. Se consideró sólo el primer hemocultivo positivo en cada paciente.

\section{Procesamiento de las muestras}

Las muestras de sangre para el examen de hemocultivo se obtuvo siguiendo el procedimiento habitual $^{27}$. La identificación microbiológica se realizó mediante pruebas bioquímicas convencionales ${ }^{28,29}$ y el estudio de sensibilidad de acuerdo a National Committee for Clinical Laboratory Standards Guideline ${ }^{30}$. El hemocultivo para anaerobios se realizó a solicitud del médico tratante.

\section{Seguimiento de pacientes}

El seguimiento intrahospitalario fue realizado por un equipo médico entrenado en las definiciones utilizadas y se constató el momento del fallecimiento, del alta o de la pérdida de seguimiento de los pacientes con bacteriemia verdadera. Las co-variables registradas fueron: datos demográficos; género, edad, primer servicio de hospitalización; datos clínicos y de laboratorio; tipo de bacteriemia, enfermedades subyacentes, uso de catéter urinario permanente, catéter venoso periférico, nutrición parenteral, catéter de hemodiálisis, quimioterapia, fiebre, shock, hemograma, proteína $\mathrm{C}$ reactiva (PCR), foco de origen, tratamiento antimicrobiano empírico; datos microbiológicos; tinción de gram, especie y/o género identificado y susceptibilidad antimicrobiana.

\section{Definiciones}

Se definió bacteriemia verdadera al aislamiento en al menos un hemocultivo de patógenos habituales como por ejemplo S. aureus, enterobacterias, $P$. aeruginosa, S. pneumoniae o bien al aislamiento de microorganismos que contaminan habitualmente los hemocultivos en al menos dos hemocultivos consecutivos, por ejemplo Staphylococcus coagulasa negativo, Streptococcus del grupo viridans, Corynebacterium spp, Bacillus spp, Propionibacterium 
acnes y algunas especies de Clostridium, en un paciente con un cuadro clínico compatible ${ }^{31}$.

Se definió bacteriemia extrahospitalaria o adquirida en la comunidad cuando fue detectada dentro de las primeras $48 \mathrm{~h}$ de hospitalización, no mediando durante ese período ninguna actividad asistencial que pueda haberla inducido y bacteriemia intrahospitalaria o nosocomial cuando fue adquirida en el hospital por un paciente internado por una razón distinta a la infección del torrente sanguíneo o si esta ocurrió en el período de 48-72 h después del alta ${ }^{24}$.

Para efectos de la investigación se definió terapia empírica inapropiada cuando una infección documentada microbiológicamente no fue tratada efectivamente al momento de su identificación; ausencia de agente antimicrobianos dirigida contra una clase específica de microorganismo (Ej. ausencia de terapia contra fungemia debida a Candida albicans); y/o administración de un agente antimicrobiano para el cual el microorganismo responsable de la infección fue resistente; la completa ausencia de terapia antimicrobiana de una infección microbiológicamente confirmada también se consideró inapropiada ${ }^{32}$.

No hubo intervención de los investigadores en la decisión de hospitalizar a los pacientes, en la extensión del estudio diagnóstico ni en el tratamiento indicado.

Se contó con la autorización del jefe del Servicio de Infectología y del de Medicina para la revisión de fichas clínicas.

\section{Análisis estadístico}

Para PCR y edad se estableció puntos de corte de $100 \mathrm{mg} / \mathrm{dl}$ y mayor e igual a 65 años, respectivamente, después del análisis exploratorio. Se estimó el HR e IC 95\% en forma univariada y multivariada mediante modelos semiparamétricos de sobrevida. Un $p$ value $<0,05$ fue considerado significativo. La sobrevida se presentó gráficamente a través de curvas de Kaplan-Meier. Se comparó la probabilidad de supervivencia entre grupos con test de log-rank. Se utilizó programa computacional Stata 10.0 para el análisis de los datos.

\section{Resultados}

\section{Análisis descriptivo e incidencia}

De 486 hemoculivos positivos, 310 hemocultivos se calificaron inicialmente como bacteriemia verdadera, sin embargo, 33 (10,6\%) de ellos, correspondieron a contaminación y 24 se perdieron de seguimiento ( $8 \%$ ); entonces 253 hemocultivos fueron incluidos dentro del estudio.

La incidencia de bacteriemias fue 11 por cada 1.000 egresos. El 55,3\% de las bacteriemias fueron hombres $(\mathrm{n}=140)$; la media de la edad fue de 62 años (DE 18 años, rango 15-94). Se registró un total de 93 (36,8\%) fallecidos; $28,2 \%$ cuando el origen fue extrahospitalario y $41,6 \%$ cuando el origen fue intrahospitalario; $17(6,7 \%)$ presentaron bacteriemia polimicrobiana. La letalidad a los 28 días de hospitalización fue de $22 \%$ y varió según el agente etiológico: Candida spp 75\%(6/8), Enterococcus spp 56,3\% (9/16), Pseudomona spp 66,7\% (10/15), Proteus spp 57,1\% (4/7), Acinetobacter spp 50\% (8/16), Klebsiella spp 40,6\% (13/32), Staphylococcus spp 36,7\% (25/68) Streptococcus spp 33,3\% (6/18), E. coli 13,7\% (7/51), Bulkordelia spp $12,5 \%$ (1/8). El servicio clínico donde se registró la mayor letalidad fue Unidad de Cuidados Intensivos 57,8\% seguido del Servicio de Urgencia 37,7\% y de Unidad de Tratamiento Intermedio 34,8\%. El 41,2\% de los pacientes con bacteriemia polimicrobiana fallecieron(7/17). La letalidad general intrahospitalaria fue mayor en mujeres 49,2\% (34/69) que hombres $35,9 \%$ (33/92) y cuando el tratamiento antimicrobiano empírico fue inapropiado 53,6\% (35/63) versus 30,5\% (58/190).

El tiempo medio entre el ingreso y la bacteriemia fue de 13,75 días (0-181 días) y fue mayor en pacientes que fallecieron 18,50 vs 10,98 días

\section{Variables que afectan la sobrevida}

En el análisis univariado, edad igual o mayor a 65 años (IC 95\% 1,28; 3,09 ), sexo femenino (IC $95 \% 1,01 ; 2,35$ ), servicio de urgencia (IC 95\% $2,01 ; 38,15)$ y unidad de cuidados intensivos (IC 95\% 1,09; 19,81), días a la bacteriemia (IC 95\% 0,$97 ; 0,99$ ), origen respiratorio (IC 95\% 1,55; 6,43) y no precisado (IC 95\% 1,23; 5,11), requerimiento de ventilación mecánia (IC 95\% 1,31; 3,04), shock (IC 95\% 1,55; 3,60), bacteriemia persistente (IC 95\% 1,07; 3,19), elevación de PCR sobre $100 \mathrm{mg} /$ dl (IC 95\% 1,29; 4,04) y tratamiento empírico inapropiado (IC 95\% 1,26; 3,01) se asociaron a mayor mortalidad, mientras que antecedente de procedimiento quirúrgico (IC 95\% 0,32; 0,82) se asoció a mayor sobrevida. No se encontró asociación entre el tipo de bacteria aislada y sobrevida (Tabla 1). 
Tabla 1. Probabilidad de muerte en pacientes con bacteriemia. Modelo univariado

\begin{tabular}{|c|c|c|c|c|c|}
\hline \multirow[t]{2}{*}{ Variable } & \multirow[t]{2}{*}{ Fallecidos \% } & \multirow[t]{2}{*}{ Total } & \multicolumn{3}{|c|}{ Modelo univariado } \\
\hline & & & HR & P value & IC $95 \%$ \\
\hline Total & 36,8 & 253 & & & \\
\hline Hombre & 33,7 & 140 & 1 & - & - \\
\hline Mujer & 58,2 & 113 & 1,54 & $<0,005$ & 1,$01 ; 2,35$ \\
\hline Edad $<65$ años & 25,8 & 132 & 1 & - & - \\
\hline Edad $>=65$ años & 48,8 & 121 & 1,98 & $<0,005$ & 1,$28 ; \quad 3,09$ \\
\hline Bacteriemia intrahospitalaria & 41,6 & 161 & 1 & - & - \\
\hline Bacteriemia extrahospitalaria & 28,3 & 92 & 0,64 & NS & 0,$39 ; \quad 1,04$ \\
\hline \multicolumn{6}{|l|}{ Servicio } \\
\hline Cirugía & 11,8 & 17 & 1 & - & - \\
\hline Urgencia & 37,7 & 61 & 8,75 & $<0,005$ & 2,$01 ; 38,15$ \\
\hline Medicina & 34,7 & 78 & 3,28 & NS & 0,$77 ; 13,90$ \\
\hline UTI & 34,8 & 23 & 4,24 & NS & 0,$90 ; 20,03$ \\
\hline $\mathrm{UCl}$ & 57,5 & 40 & 4,64 & $<0,005$ & 1,$09 ; 19,81$ \\
\hline Otros & 25,8 & 31 & 3,48 & NS & 0,$73 ; 16,50$ \\
\hline Días a bacteriemia & 18,5 & $0-182$ & 0,98 & $<0,005$ & 0,$97 ; \quad 0,99$ \\
\hline \multicolumn{6}{|l|}{ Foco } \\
\hline Urinario & 18,7 & 64 & 1 & - & - \\
\hline No precisado & 53,2 & 47 & 2,51 & $<0,005$ & 1,$23 ; \quad 5,11$ \\
\hline Respiratorio & 56,7 & 37 & 3,15 & $<0,005$ & 1,$55 ; \quad 6,43$ \\
\hline Catéter & 30,0 & 60 & 1,11 & NS & 0,$53 ; \quad 2,34$ \\
\hline Digestivo & 50,0 & 20 & 2,32 & NS & 0,$99 ; \quad 5,39$ \\
\hline Quirúrgico & 20,0 & 10 & 1,22 & NS & 0,$27 ; \quad 5,47$ \\
\hline Piel y partes blandas & 50,0 & 8 & 2,14 & NS & 0,$69 ; \quad 6,66$ \\
\hline \multicolumn{6}{|l|}{ Procedimientos invasivos } \\
\hline Cirugía & 32,2 & 84 & 0,52 & $<0,005$ & 0,$32 ; \quad 0,82$ \\
\hline Ventilación mecánica & 65,6 & 67 & 1,99 & $<0,005$ & 1,$31 ; 3,04$ \\
\hline Catéter urinario & 47,3 & 127 & 1,75 & NS & 1,$13 ; \quad 2,72$ \\
\hline Catéter venoso central & 49,5 & 97 & 1,48 & NS & 0,$97 ; \quad 2,25$ \\
\hline Catéter de hemodiálisis & 45,4 & 64 & 1,13 & NS & 0,$72 ; \quad 1,77$ \\
\hline Catéter de nutrición parenteral & 52,5 & 40 & 0,81 & NS & 0,$49 ; \quad 1,35$ \\
\hline \multicolumn{6}{|c|}{ Factores clínicos predisponentes } \\
\hline Diabetes & 38,0 & 79 & 0,84 & NS & 0,$53 ; \quad 1,32$ \\
\hline Insuficiencia renal crónica & 32,3 & 62 & 0,79 & NS & 0,$48 ; 1,31$ \\
\hline Daño hepático crónico & 50,0 & 18 & 1,50 & NS & 0,$75 ; \quad 2,99$ \\
\hline Cáncer & 53,9 & 26 & 1,50 & NS & 0,$75 ; \quad 2,99$ \\
\hline Inmunosupresión & 28,4 & 21 & 0,61 & NS & 0,$24 ; \quad 1,51$ \\
\hline Neutropenia & 57,2 & 14 & 1,37 & NS & 0,$63 ; \quad 2,97$ \\
\hline Quimioterapia & 40,0 & 5 & 1,21 & NS & 0,$16 ; 8,75$ \\
\hline
\end{tabular}


Tabla 1. Continuación

\begin{tabular}{|lrrrcc|}
\hline Variable & Fallecidos $\%$ & Total & \multicolumn{2}{c|}{ Modelo univariado } \\
IC95\%
\end{tabular}

Tabla 2. Probabilidad de muerte en pacientes con bacteriemia. Modelo univariado

\begin{tabular}{|lccccc|}
\hline Variable & Fallecidos $\%$ & Total & \multicolumn{3}{c|}{ Modelo multivariado } \\
Edad $>=65$ años & 48,8 & 121 & HR & P value & IC 95\% \\
Mujer & 58,2 & 113 & 1,44 & $<0,005$ & 1,$49 ; 3,99$ \\
\hline Cirugía & 32,2 & 84 & 0,42 & $<0,005$ & 1,$05 ; 2,70$ \\
\hline Shock & 69,2 & 68 & 2,97 & $<0,005$ & 0,$24 ; 0,72$ \\
\hline PCR $>=100 \mathrm{mg} / \mathrm{dl}$ & 41,6 & 149 & 2,10 & $<0,005$ & 1,$85 ; 4,76$ \\
\hline
\end{tabular}

Cuando se realizó el análisis multivariado de estas variables, edad mayor de 65 años (IC 95\% 1,$42 ; 3,77$ ), sexo femenino (IC $95 \% 1,06 ; 2,71$ ), shock (IC 95\% 2,01; 5,22) y PCR mayor de 100 $\mathrm{mg} / \mathrm{dl}$ (IC 95\% 1,$17 ; 3,78)$ se asociaron significativamente a mayor mortalidad, mientras que procedimientos quirúrgicos (IC 95\% 0,25; 0,75) durante la hospitalización se correlacionó con menor mortalidad (Tabla 2).

En las Figuras 1-5 se presenta como cerca de $2 / 3$ de la cohorte se encuentre viva al día 28 de hospitalización y como la probabilidad de sobrevida desciende significativamente en mujeres, en aquellos que presenta shock, en los mayores de 65 años y en aquellos con PCR igual o mayor a $100 \mathrm{mg} / \mathrm{dl}$. 


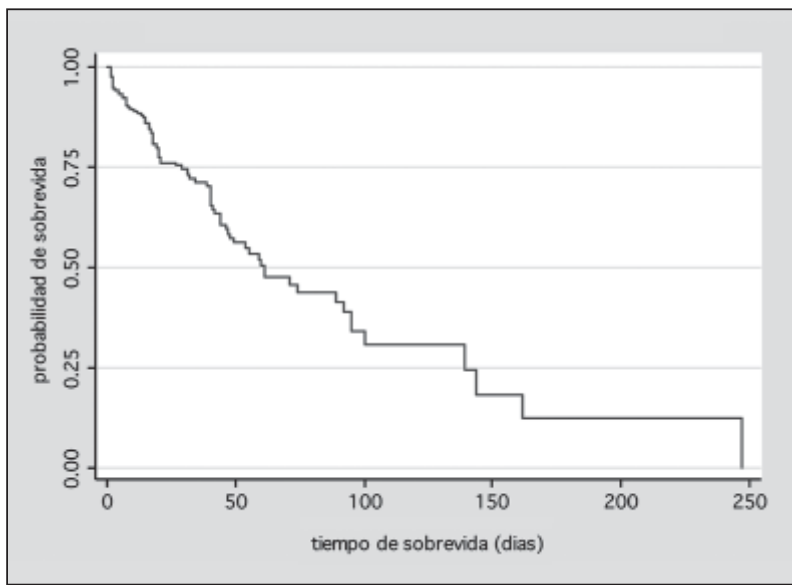

Figura 1. Curva de Kaplan-Meier de la probabilidad de sobrevida de la cohorte de pacientes con bacteriemia.

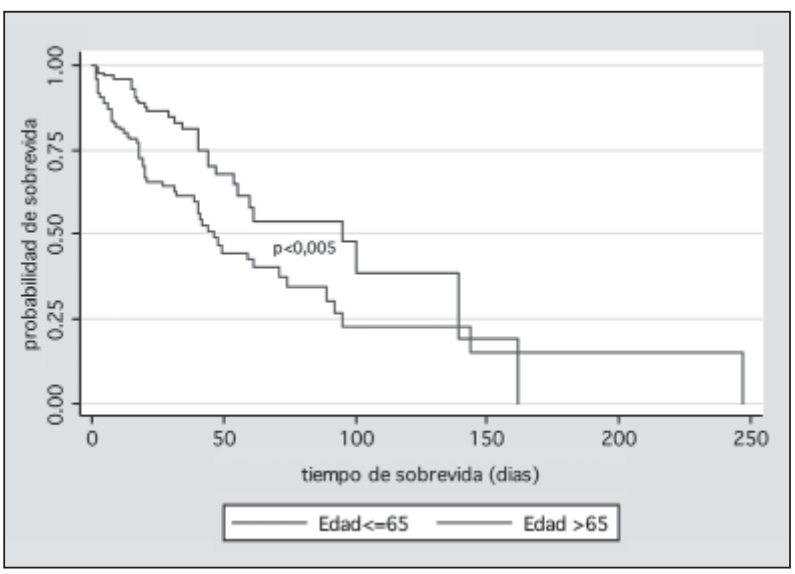

Figura 2. Curva de Kaplan-Meier según edad.



Figura 3. Kaplan Meier según PCR.

\section{Discusión}

En Chile existe escasa información de las características clínico-microbiológicas de las bacteriemias de origen nosocomial y de las adquiridas en la comunidad; los datos en nuestro país son en general limitados a las bacteriemias que se vigilan por normativa ministerial, por tal motivo, los estudios epidemiológicos globales y prospectivos de bacteriemia son útiles para conocer la etiología y los factores pronósticos en un entorno concreto y detectar aquellos factores que puedan ser modificables.

La tasa de incidencia de bacteriemias en nuestro hospital fue de 11 por 1.000 egresos, lo que se encuentra en el rango de lo reportado a nivel internacional (5-28 episodios/1.000 pacientes) ${ }^{11-13}$.

La letalidad de $22 \%$ a los 28 días de hospitalización es similar a lo publicado. Bou reportó una letalidad de $18 \%$, Pazos $22 \%$, Wisplinghoff $27 \%$ y Gatell $18 \%$. Sin embargo, Valles reportó una letalidad de $41 \%$ en pacientes admitidos en $\mathrm{UCI}^{12-14,33}$. Estos resultados deben valorarse en relación a criterios de gravedad de los pacientes atendidos. Nuestro estudio fue diseñado para estimar la letalidad cruda y no la atribuible dada la cantidad de factores que pueden influir en esta última definición.

Los factores pronósticos que se repiten con mayor frecuencia en la literatura son edad, shock séptico, APACHE II $>15$, foco indeterminado y no urinario, neoplasia, neutropenia y el tratamiento antibiótico empírico inadecuado ${ }^{11-15}$. Sin embargo, a diferencia de estos autores nuestro estudio encontró que aquellos pacientes sometidos a cirugía presentaron menor mortalidad, posiblemente porque estos sujetos se encuentraron bajo una atención especial por parte del equipo médico antes, durante y después del procedimiento invasivo. En contraposición, edad igual o mayor a 65 años, sexo femenino y PCR igual o mayor a $100 \mathrm{mg} / \mathrm{dl}$, se asociaron a mayor mortalidad. Un estudio dirigido podría revelar una interesante utilidad de esta última variable, en la construcción de un score pronóstico. En este estudio no se logró determinar una asociación significativa 


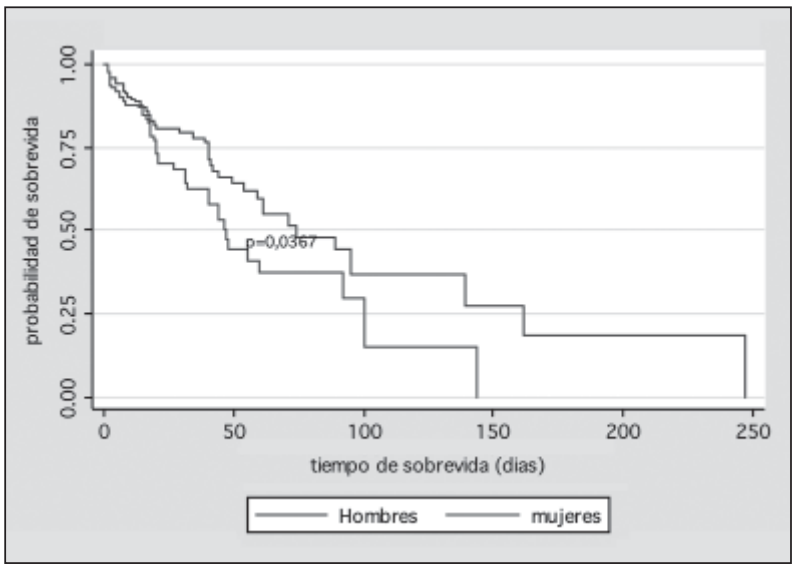

Figura 4. Kaplan Meier según sexo.

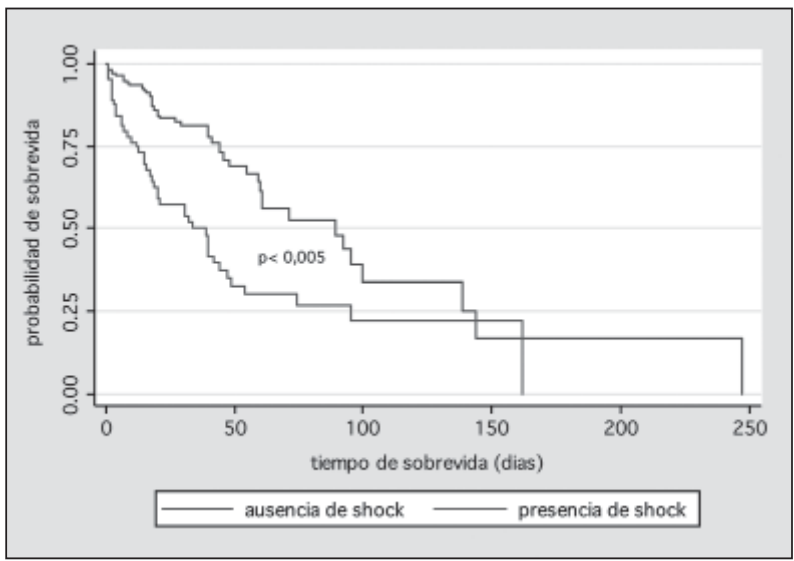

Figura 5. Kaplan-Meier según shock.

entre tratamiento antimicrobiano inapropiado y mortalidad intrahospitalaria, posiblemente por el tamaño de la muestra. La edad y el shock se repiten en nuestro estudio como factores de riesgo de menor sobrevida lo que es concordante con la literatura y nos obliga a prestar especial atención a pacientes mayores así como a su condición hemodinámica de los pacientes a su ingreso.

Limitaciones propias de la falta de seguimiento extrahospitalario y el sesgo derivado de la inclusión de pacientes que acuden a un centro de atención de salud impiden la extrapolación de los resultados a la población general.

\section{Referencias}

1. Moreno R, Afonso S, Fevereiro T. Incidence of sepsis in hospitalized patients. Curr Infect Dis Rep 2006; 8: 346-50.

2. Ruiz-Giardin JM, Noguerado Asensio A. [Bacteremia]. An Med
Interna 2005; 22: 105-7.

3. Panceri ML, Vegni FE, Goglio A, Manisco A, Tambini R, Lizioli A, et al. Aetiology and prognosis of bacteraemia in Italy. Epidemiol Infect 2004; 132: 647-54.

4. Wisplinghoff H, Bischoff T, Tallent SM, Seifert H, Wenzel RP, Edmond MB. Nosocomial bloodstream infections in US hospitals: analysis of 24,179 cases from a prospective nationwide surveillance study. Clin Infect Dis 2004; 39: 309-17.

5. Brun-Buisson C, Doyon F, Carlet J. Bacteremia and severe sepsis in adults: a multicenter prospective survey in ICUs and wards of 24 hospitals. French Bacteremia-Sepsis Study Group. Am J Respir Crit Care Med 1996; 154: 617-24.

6. Matas L, Marti C, Morera MA, Sierra M, Vilamala A, Corcoy F. [Bacteremia in 13 general hospitals of the province of Barcelona. Prospective study of 1,674 episodes. Group of Microbiologists of the County Hospitals of Catalonia]. Enferm Infecc Microbiol Clin 1995; 13: 345-55.

7. Bouza E, Pérez-Molina J, Muñoz P, (ESGNI) aCGotESGoNI. Report of ESGNI-001 and ESGNI-002 studies. Bloodstream infections in Europe. Clin Microbiol Infect 1999; 5: 2S1-2S12.

8. Luzzaro F, Ortisi G, Larosa M, Drago M, Brigante G, Gesu G. Prevalence and epidemiology of microbial pathogens causing bloodstream infections: results of the OASIS multicenter study. Diagnostic Microbiology and Infectious Disease 2011; 69: 363-9.

9. Friedman ND, Kaye KS, Stout JE, McGarry SA, Trivette SL, Briggs JP, et al. Health care-associated bloodstream infections in adults: a reason to change the accepted definition of communityacquired infections. Ann Intern Med 2002; 137: 791-7.

10. Son JS, Song JH, Ko KS, Yeom JS, Ki HK, Kim SW, et al. Bloodstream infections and clinical significance of healthcare-associated bacteremia: a multicenter surveillance study in Korean hospitals. J Korean Med Sci; 25: 992-8.

11. Javaloyas M, García-Somoza D, Gudiol F. Epidemiology and prognosis of bacteremia: a 10-y study in a community hospital. Scand J Infect Dis 2002; 34: 436-41.

12. Pazos Anon R, Fernández Rodríguez R, Paz Vidal I, Tinajas A, Canton I, Abel V, et al. [Prognostic factors of bacteremia: prospective study]. An Med Interna 2001; 18: 415-20.

13. Mylotte JM, Tayara A, Goodnough S. Epidemiolo- 
gy of bloodstream infection in nursing home residents: evaluation in a large cohort from multiple homes. Clin Infect Dis 2002; 35: 1484-90.

14. Lizaso D, Aguilera CK, Correa M, Yantorno ML, Cuitino M, Pérez L, et al. [Nosocomial bloodstream infections caused by gram-negative bacilli: epidemiology and risk factors for mortality]. Rev Chilena Infectol 2008; 25: 368-73.

15. Corredoira Sánchez JC, Casariego Vales E, Alonso García P, Coira Nieto A, Varela Otero J, López Álvarez MJ, et al. [Bacteremia in the elderly. Clinical features and prognostic factors]. Med Clin (Barc) 1997; 109: 165-70.

16. Kollef MH, Ward S, Sherman G, Prentice D, Schaiff $\mathrm{R}$, Huey $\mathrm{W}$, et al. Inadequate treatment of nosocomial infections is associated with certain empiric antibiotic choices. Crit Care Med 2000; 28: 3456-64.

17. Wyllie DH, Crook DW, Peto TE. Mortality after Staphylococcus aureus bacteraemia in two hospitals in Oxfordshire, 1997-2003: cohort study. BMJ Clinical Research Ed 2006; 333: 281.

18. Abhilash KP, Veeraraghavan B, Abraham OC. Epidemiology and outcome of bacteremia caused by extended spectrum beta-lactamase (ESBL)-producing Escherichia coli and Klebsiella spp. in a tertiary care teaching hospital in south India. J Assoc Physicians India 2010; 58 Suppl: 13-7.

19. Martin GS, Mannino DM, Eaton S, Moss M. The epidemiology of sepsis in the United States from 1979 through 2000. New Engl J Med 2003; 348: 1546-54.

20. Wisplinghoff $H$, Seifert H, Wenzel RP, Edmond MB. Current trends in the epidemiology of nosocomial bloodstream infections in patients with hematological malignancies and solid neoplasms in hospitals in the United States. Clin Infect Dis 2003; 36: 1103-10.

21. Dalton HP, Allison MJ. Etiology of bacteremia. Appl Microbiol 1967; 15: 808-14.

22. Weinstein MP, Towns ML, Quartey SM, Mirrett S, Reimer LG, Parmigiani G, et al. The clinical significance of positive blood cultures in the 1990s: a prospective comprehensive evaluation of the microbiology, epide- miology, and outcome of bacteremia and fungemia in adults. Clin Infect Dis 1997; 24: 584-602.

23. Pohlenz M BP, Pidal P, Bustamante R. Informe de las vigilancias intrahospitalarias. Chile. Ministerio de Salud 2006; 1-57.

24. Cisneros-Herreros JM, Cobo-Reinoso J, Pujol-Rojo M, Rodríguez-Bano J, Salavert-Lleti M. [Guidelines for the diagnosis and treatment of patients with bacteriemia. Guidelines of the Sociedad Espanola de Enfermedades Infecciosas y Microbiología Clinica]. Enfermedades Infecc Microbiol Clin 2007; 25: 111-30.

25. Sifuentes-Osornio J, Guerrero-Almeida MC, Ponce de León-Garduno LA, Guerrero-Almeida ML. [Trends for bacteremia and risk factors for death in a tertiary hospital in Mexico City. 1981-1992]. Gaceta Médica de México 2001; 137: 191-202.

26. Garner JS, Jarvis WR, Emori TG, Horan TC, Hughes JM. CDC definitions for nosocomial infections, 1988. American Journal of Infection Control 1988; 16: 128-40.

27. Wilson M, Mitchell M, Morris A, Murray P, Reimer L, Reller L, et al. Principles and Procedures for Blood Cultures; Approved Guideline 2007; 27: 4-15.

28. Baron EJ. Cumitech 1C: Blood Cultures IV. In: ASM Press; 2005.

29. Murray PR, Baron EJ, Pfaller MA, Tenover FC, Yolken RH. Manual of Clinical Microbiology. 7 th ed; 1999.

30. Wikler Mathew CF, Busk K, Dudley M, Eliopoulus G, Hardy D, Hindler J, et al. Performance Standards for Antimicrobial Disk Susceptibility Test; Aproved StandardTenth Edition. Clinical and Laboratory Standard Institute 2009; 29.

31. Bates DW, Lee TH. Rapid classification of positive blood cultures. Prospective validation of a multivariate algorithm. JAMA 1992; 267: 1962-6.

32. Kollef $\mathrm{MH}$. Inadequate antimicrobial treatment: an important determinant of outcome for hospitalized patients. Clin Infect Dis 2000; 31 Suppl 4: S131-8.

33. García Ordoñez MA, Colmenero Castillo JD. [Prognostic factors in bacteremia and sepsis]. An Med Interna 2006; 23: 53-5. 